\title{
High-overtone Bulk Acoustic Resonator (HBAR) as passive sensor: towards microwave wireless interrogation
}

\author{
J.-M Friedt, N. Chrétien \\ SENSeOR \\ Besançon, France \\ Email: jmfriedt@femto-st.fr
}

\author{
T. Baron, É. Lebrasseur, G. Martin, S. Ballandras \\ FEMTO-ST Institute, UMR 6174 \\ 32 avenue de l'observatoire, 25044 Besançon, France \\ Email: ballandr@femto-st.fr
}

\begin{abstract}
Increasing the operating frequency of wireless passive sensors is suitable for reducing the global sensor size currently dominated by antenna dimensions, and for improved directivity of the probe electromagnetic signal with either electronic or mechanical beam sweeping by the reader for space domain multiplexing. While surface acoustic wave (SAW) transducers patterned on piezoelectric substrate are one of the standard approach for passive wireless sensing, their operating frequency is limited by lithography resolution. One alternative approach to increase the operating frequency is to exploit bulk acoustic resonators operating at high overtone - the so-called HBARs. In this paper, the interrogation of such devices is demonstrated either in the time domain (delay line) or frequency domain (resonator), taking advantage of their spectral characteristics, i.e. a comb of modes in the frequency domain, or a comb of time-domain reflections.

Index Terms - wireless, sensor, high overtone bulk acoustic resonator, HBAR, microwave, RADAR
\end{abstract}

\section{INTRODUCTION}

Acoustic sensors are designed either as narrowband, frequency-defined transducers, following resonator considerations, or as wide-band, time-domain pulse propagation delay defined, delay lines. The design considerations for such devices are rather different: high quality factor for resonators in order to take advantage of the long energy decay of the unloading resonator to differentiate the acoustic sensor response from electromagnetic clutter ; high coupling for delay lines in order to maximize the electromagnetic to mechanical conversion through piezoelectric effects (reverse and direct). The linear process of piezoelectric conversion yields significantly improved performances for acoustic transducers acting as passive sensors probed through a wireless link with respect to silicon based Radio-Frequency IDentification (RFID) tags: while the latter require a minimum amount of incoming energy for the voltage at the rectifier level to reach the diode threshold voltage - limiting the interrogation range
[1] to contact in the lower radiofrequency range and to a few tens of centimeters in the Ultra-Hight Frequency (UHF) range - acoustic sensors will always generate a return signal, and the interrogation range is solely limited by the receiver noise level below which the received signal is no longer detectable. Furthermore, RFID chips are most commonly used for storing an identifier - a series of fixed bit values to be returned by antenna impedance modulation by polarizing a transistor on a coil to which the silicon chip is attached - and the additional power consumption which would be associated with the analog to digital conversion process needed for measuring a physical quantity is hardly ever considered in assessing the interrogation range of silicon RFID. On the other hand, the short energy storage duration of acoustic sensors - typically $5 \mu$ s for either delay line or resonator configurations - significantly restricts the signal processing schemes for improving the signal to noise ratio of the reception stage through the classical averaging or cross-correlation strategies (as used for example in Global Positioning Satellite - GPS - when cross correlating long pseudo-random bit sequences do detect a signal below the thermal noise level), hence limiting the interrogation to a few meters.

Although a bistatic antenna, switched RADAR interrogation strategy for probing acoustic sensor response allows for clearly distinguishing both types of devices delay line or resonators - the need for optimally coupled resonators to minimize conversion losses during wireless interrogation must be weighted against the reduced insertion loss delay lines to optimize the energy balance in the interrogation process. For most wireless applications using passive radio-frequency (RF) devices, surface acoustic waves (SAW) have been preferred as they allow for an accurate definition of operating frequencies and for shaping their spectral response according to specific 
user requirements. By the way, SAW delay lines and resonators must be specifically used with the proper interrogation electronics and thus do not reveal versatile enough to answer time and frequency domain interrogation scheme at once.

High-overtone Bulk Acoustic Resonators (HBAR) consist of a stack of a highly coupled piezoelectric layer deposited or bonded atop a low loss propagation substrate. This kind of devices has been classically considered for their capability to excite high quality factor resonating modes. Contrarily to Film Bulk Acoustic Resonator consisting of a metalthin filmmetal architecture, the above-described HBAR structure provides significant ruggedness to the resonator, however sacrificing the typical single-mode signature of FBAR. The spectral response of HBAR actually consists of a comb of modes in the frequency domain, thus yielding a reciprocal comb of time-domain reflections according Fourier principles.

In this document, we consider HBARs not as frequency combs but as time-domain reflector combs. The interest of these transducers is demonstrated here for wireless sensor interrogation, either interrogating a specific mode in the spectral domain, particularly meeting Instrument-Scientific-Medical (ISM) regulations for various frequencies $(434,868,915 \mathrm{MHz}$ or even $2.45 \mathrm{GHz}$ ) or in time domain, taking advantage of their periodic time response. Furthermore, the bulk nature of these devices provides ruggedness to the sensor and therefore allows for multiple applications (temperature, pressure, gravimetric sensors). The remarkable versatility of these devices is particularly illustrated in this work for temperature measurements (Fig. 1) [2].

\section{ACOUSTIC TRANSDUCERS AS PASSIVE COOPERATIVE TARGETS}

By getting rid of the thin interdigitated transducers (IDT) prone to large resistive losses when patterned over highly coupled materials (due to high current circulation, particularly when operating near or above $500 \mathrm{MHz}$ ), the HBAR geometry provides the means of reaching high quality factors even when using highly coupled single piezoelectric substrates such as lithium niobate. Furthermore, removing the lithography resolution limitation (which is replaced by the ability to manufacture the thin single piezoelectric layer above a low loss substrate - either by deposition or assembly followed by grinding and polishing [3]) opens the ability to reach frequency well beyond those classically reached by surface acoustic wave transducers using research grade clean-room facilities.

For frequency source applications, a high quality factor of the considered acoustic modes is mandatory
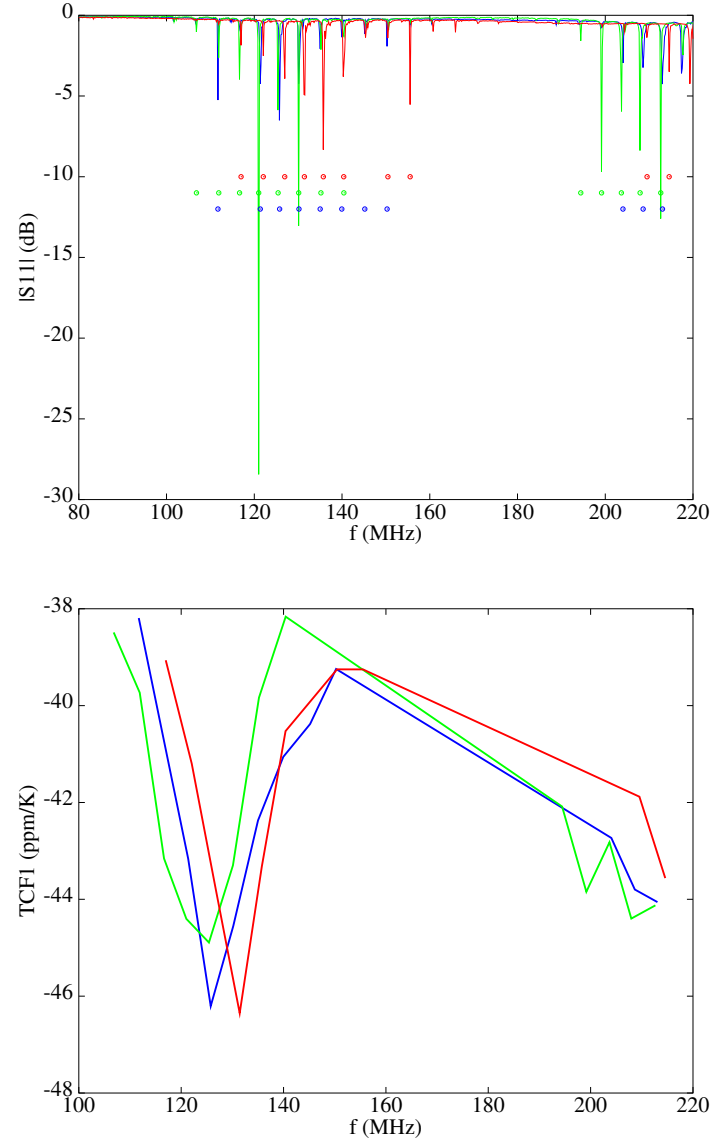

Fig. 1. Top: $\left|S_{11}\right|$ spectra of three HBARs made of a thin lithium niobate piezoelectric layer over quartz. Bottom: first order temperature coefficient of frequency as a function of temperature for various overtones identified as circles on the top graph.

as it defines the coupling capability of the device with the incoming electromagnetic energy and the energy storage to energy loss for each period, and thus the filtering capability of the resonator when subject to external noise. The specific requirements for wireless sensor applications are not that stringent. Indeed, while an oscillator characteristics is directly related to the quality factor of the resonator in the feedback loop (phase noise characteristics and Leeson frequency position), a wireless sensor application only requires that the energy loading lasts longer than the environmental clutter so that the RADAR receiver can discriminate between passive reflectors (clutter) and the sensor response. On the other hand, in a delay line approach, the coupling coefficient $k^{2}$ is a fundamental characteristic considering the definition of the conversion capability from an incoming electromagnetic wave to the acoustic 
wave storing energy in the sensor and vice versa. As a consequence, this parameter must be related to the use of HBAR as wireless sensors and the corresponding condition has to be complied with.

While the wide frequency operating conditions of HBAR offers advantage over other schemes compatible with only a single RADAR unit operating around a given carrier frequency, the interpretation of the recorded resonance frequency or time delay as a function of physical quantity is a complex issue due to the dependence of the effect of the physical quantity with overtone number. Indeed, a given physical quantity (e.g. temperature effect on material constants) will generate an observed velocity change of the acoustic wave (either as a time delay or frequency) dependent on the energy distribution in the active thin piezoelectric film and the thicker low acoustic loss substrate. Since both materials are usually different, the velocity relation to the physical quantity is dependent on the overtone number, as experimentally observed for the Temperature Coefficient of Frequency (TCF) of a lithium niobate over quartz HBAR (Fig. 1). Thus, a complete characterization of all the needed overtone as a function of environmental effects is needed for an accurate interpretation of the measurement results.

As an example of a wireless measurement of an HBAR transducer acting as a passive sensor interrogation by a commercial Ground Penetrating RADAR (GPR) unit (Malå unit fitted with a shielded $250 \mathrm{MHz}$ antenna), Fig. 2 exhibits the radargram as two sensors are located under the antenna radiation pattern. Two different HBAR transducers were used in this experiment, one exhibiting a significant coupling at the operating frequency (blue) and another one whose main coupling (as defined by the thin piezoelectric active layer thickness) yields modes above the operating frequency. Such broadband device were suited as well for measurement when using unshielded 100 and $200 \mathrm{MHz}$ antennas, emphasizing the compatibility of a single sensor with multiple operating frequencies as selected when adjusting for electromagnetic penetration depth as a function of moisture level and soil property. Indeed, the lower the operating frequency, the deeper the penetration depth of the electromagnetic wave but the poorer the spatial resolution. Here, we demonstrate that a single sensor is compatible with a wide range of antennas, with no modification of the GPR other than software post-processing for phase identification of the time delay of successive pulses [4]. The issue of the different sensitivity of the various overtones as a function of the physical quantity (here temperature) means that the dependence of each overtone with the measured quantity must be calibrated before
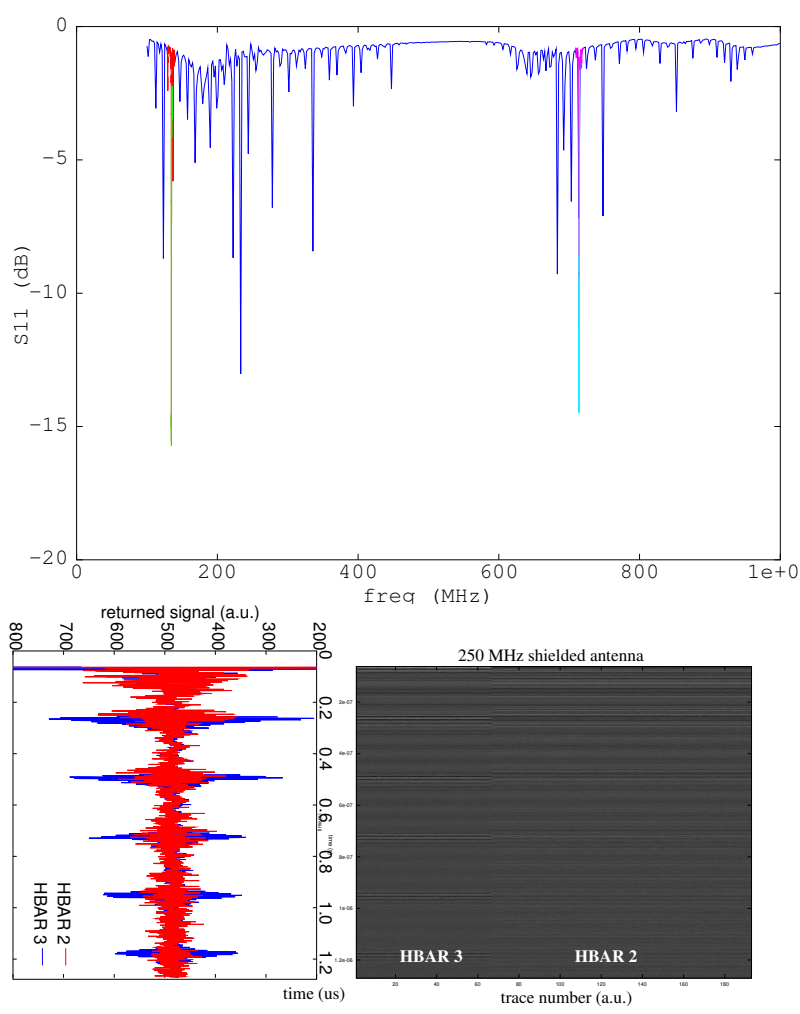

Fig. 2. Top: frequency domain transfer function of a HBAR. Notice the broad set of resonances ranging from 100 to $250 \mathrm{MHz}$, and the second set of resonances around $700 \mathrm{MHz}$. The colored modes around $130 \mathrm{MHz}$ and $700 \mathrm{MHz}$ are measurements on a narrower frequency span in order to better resolve the resonance and avoid measurement aliasing by using too broad frequency steps. Bottom: delay line-like responses of two HBAR sensors probed by a $250 \mathrm{MHz}$ shieldedantenna Malå ground penetrating RADAR unit. Two HBAR were sequentially located under the shielded antenna unit. The left graph is a A-scan cross section of the radargram, as each of the sensor returned signals are visible with a time interval defined by the low-acoustic loss substrate and the bulk acoustic wave velocity in the substrate.

deployment of the sensor in the field when the operating frequency of the RADAR is not known before the measurement is performed.

The energy balance in a wireless link budget is thus given by the antenna efficiency, the propagation loss and the coupling coefficient, the latter value being considered, in a RADAR equation, as part of the RADAR cross section of the sensor subjected to the incoming electromagnetic wave irradiation.

These considerations strengthen the above arguments for the HBAR as an attractive combination of a highly coupled transducer together with loss-less propagation and sharp acoustic reflections thus providing quite long mode ringing even if interrogated with short interrogation pulse. Moreover, the need for clearly separating the 
numerous back-and-forth acoustic contributions imposes rules on the delay of the excitation pulse: the number of sine-waves composing this pulse must be smaller than the number of wavelength of the lowest mode excited in the interrogation sequence along the HBAR thickness.

\section{MicROWAVE RADAR COMPATIBILITY}

Although a wide range of RADARs operate in the HF and VHF bands - especially when long range is needed - multiple configurations operate in the microwave range as well [5], [6]. In [7], the requirement of the lack of local energy source on the target sensor (in their case a passive microwave reflector trihedrons (so called "passive markers") and all weather operation emphasizes the advantages of the use of a microwave RADAR (18$26 \mathrm{GHz}$ frequency-stepped RADAR operating over a $8 \mathrm{GHz}$ bandwidth) associated with cooperative targets for monitoring with sub-mm resolution landslide environments. We consider that adding physical quantity measurement (temperature, strain, pressure) would add valuable information in this context. However, with the current lithography limitations on single crystal piezoelectric substrate, the microwave range is well beyond the SAW-industry cleanroom manufacturing capability for acoustic transducers. Consequently, means of probing VHF acoustic transducers using an incoming microwave beam are welcome to be considered following the harmonic RADAR approach [8].

In order to use the same approach of a passive sensor acting as a cooperative target (as described in section II), two different approaches can be considered. On the one hand, one can use low loss dielectric substrates whose properties significantly vary with the physical quantity under investigation [9], [10], [11], on which the antenna is patterned. On the other hand, converting the incoming microwave radiation to an ultra high frequency (UHF) signal allows for storing energy in the resonator using signal modulation/demodulation techniques. The latter approach allows for the use of the sensors described in section II, with the drawback of the inefficient non-linear conversion process by a diode, as is well known from the field on electromagnetic energy harvesters [12], [13].

Nevertheless, we demonstrate the conversion from an incoming modulated $10 \mathrm{GHz}$ signal to a UHF probe signal used for probing an acoustic resonator, which then radiates the resulting VHF energy (Fig. 3). Thus, rather than using a backscatter approach in which the tag communicates by modulating the antenna impedance as the target is illuminated by the RADAR [14], we here considered loading the sensor at one frequency (the modulated microwave carrier) and detecting the returned signal propagating in one of the allocated ISM bands. We will particularly focus on the $434 \mathrm{MHz}$-centered band.

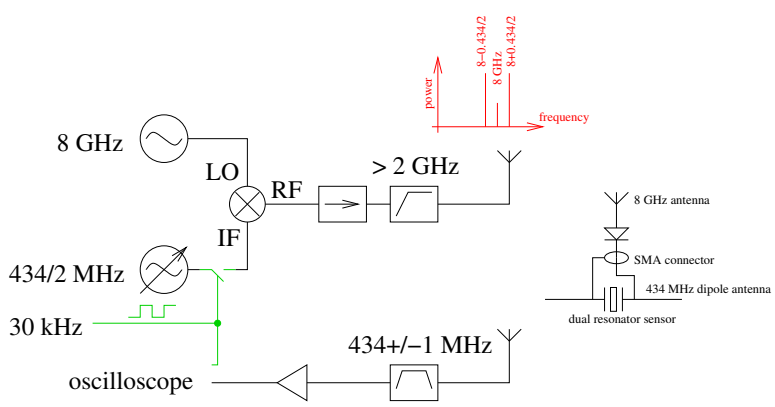

Fig. 3. Schematic of the microwave probing approach of an UHF acoustic sensor. The incoming electromagnetic wave is a $10 \mathrm{GHz}$ carrier mixed with an UHF signal swept around $434 \mathrm{MHz}$ where one of the acoustic resonances is located. When energy loading occurs, the sensor releases the stored energy at its resonance frequency and the power detector is used to monitored the returned power after a delay long enough for the clutter to fade out.

A $10 \mathrm{GHz}$ carrier signal is generated by a Wiltron 69137A: the actual carrier frequency should be selected in order to comply with ISM regulations and the availability of a demodulation diode on the sensor side. Mixing with a modulation frequency, which is swept around the $434 \pm 1 \mathrm{MHz}$ band, generates the excitation signal which is emitted to load the sensor.

On the sensor side, the diode considered for the extraction of the modulation signal from the microwave carrier is a Herotek DT8016 operating in the 8 to $16 \mathrm{GHz}$ range. Beyond the compatibility with available RADAR sources, the gain of loading the sensor at microwave frequencies is related to the high directivity of the emitted beam which provides the space-domain multiplexing capability as well as high-gain antenna designs in volumes compatible with industrial monitoring applications.

Finally, the receiver (Fig. 4) is either a radiofrequencycompatible digital oscilloscope (LeCroy WaveRunner 6200) or a spectrum analyzer displaying the power returned at the modulation frequency, which lies within one of the allocated ISM bands (here $434 \pm 1 \mathrm{MHz}$ ). In an embedded implementation of the interrogation unit, a fast (bandwidth $\geq 1 \mathrm{MHz}$ for a response time lower than $1 \mu \mathrm{s}$ ) power detector such as the Analog Devices AD8362 is used to monitor the returned power in the [433 : 435] $\mathrm{MHz}$ range as the emitted modulation frequency is swept within this range. A significant returned signal, as occurs when the modulation frequency is close to an acoustic resonance, is observed as an increase in the returned power sampled $1 \mu$ after switching off the excitation pulse. Processing the resulting transfer function allows for an identification of the resonance frequency 
and thus of the physical quantity under investigation.

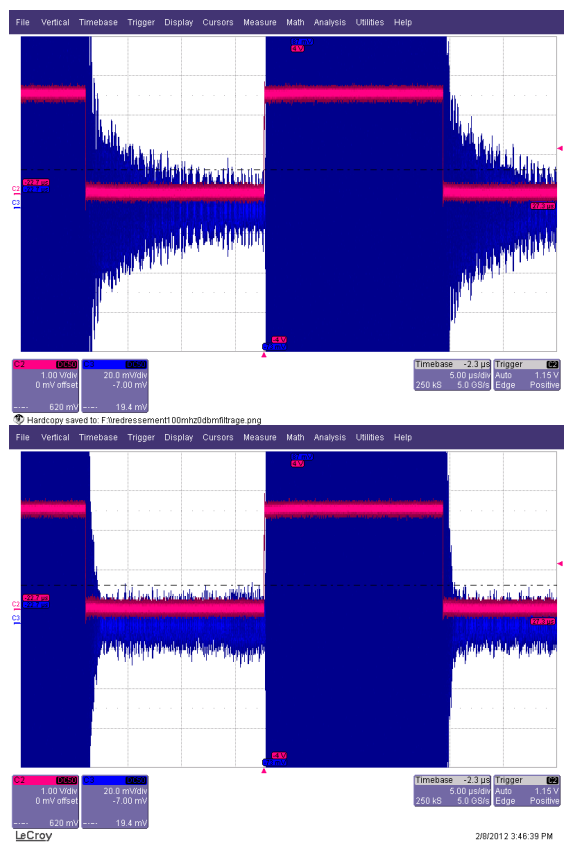

Fig. 4. Top: the power returned around $434 \mathrm{MHz}$, as the modulation signal of the $10 \mathrm{GHz}$ carrier is close to the resonance frequency of a sensor. Bottom: the returned power around $434 \mathrm{MHz}$ as the modulation signal of the $10 \mathrm{GHz}$ carrier is off resonance frequency. Notice the exponentially decaying signal after the loading pulse is switched off (7 $\mu$ s after the beginning of the graph) and lasting about $10 \mu$ s when the modulation frequency is close to a resonance (top), while only noise is visible within the $10 \mu$ s after switching off the excitation pulse in case the modulation is far from an acoustic resonance (bottom).

In a backscattering approach familiar to RFID, the power at the modulation frequency of the emitted signal (around $434 \mathrm{MHz}$ ) drops as the electromagnetic wave couples to the sensor. Hence, two approaches familiar to wireless passive sensor measurement are applicable, either a far field approach in which the returned power after clutter has dissipated is recorded (sequentially switching on and off the RADAR output), or the power at the modulation signal is recorded as a continuous wave is emitted, with a returned energy drop as a fraction of the electromagnetic field is coupled to the sensor. Both approaches are illustrated on Fig. 5, with the inset spectrum analyzer exhibiting the two dips of a dualresonator sensor characterized with two modes around 433 and $434 \mathrm{MHz}$, and the oscilloscope screen (also shown in Fig. 4) exhibiting the sensor returned signal in a pulsed mode RADAR approach. In both cases, the sensor is connected to a setup of two antennas in parallel, one being a dipole tuned to resonate around $10 \mathrm{GHz}$ (incoming excitation signal) and the other a dipole tuned to resonate below $434 \mathrm{MHz}$ (returned signal). No impedance matching consideration was performed at this early demonstration stage, although a matching network and including multiple monopoles and rectifying diode along the $434 \mathrm{MHz}$ dipole are expected to significantly improve energy transfer yield and thus interrogation range.

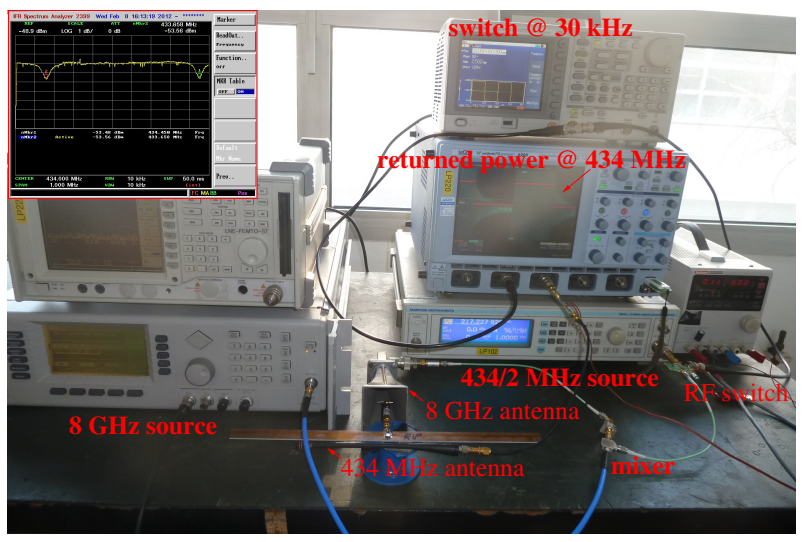

Fig. 5. Experimental setup in which both pulsed mode (monitoring the returned power from the sensor as the clutter has faded out) and continuous wave (the modulation frequency power drops as the electromagnetic field couples with the sensor at resonance) are illustrated in this setup.

Alternatively, in order to avoid the low efficiency of the diode-based demodulation and return to a linear process even at microwave frequencies, HBARs have been designed which exhibit some resonance up to $4 \mathrm{GHz}$. Such a configuration is achieved by gluing two lithium niobate layers with a thin $(\simeq 1 \mu \mathrm{m}$ thick) SU8 film (Fig. 6). However, current technological limitations yield excessive losses and thus low efficiency when interrogated through a wireless link. Fine tuning the reflection coefficient of the buried interface - here a polymeryzed organic compound, allows for designing a 3-layer HBAR in which the thin piezoelectric layer acts as the transducer, the thin polymer layer acts as the energy storage substrate with wide mode separation thanks to its micrometer range thickness, and the thick substrate below the polymer layer provides a rugged sensor configuration with no membrane or thin film which would otherwise make the whole system fragile. Tuning the acoustic impedance mismatch at both interfaces piezoelectric-polymer and polymer-substrate - allows for improving the microwave response while preventing too much energy from reaching the thick (VHF-UHF modes) substrate. An inorganic interface layer other than a polymer will certainly improve the mode resolution by inducing lower acoustic losses and improved mode confinement (resonator cavity). 


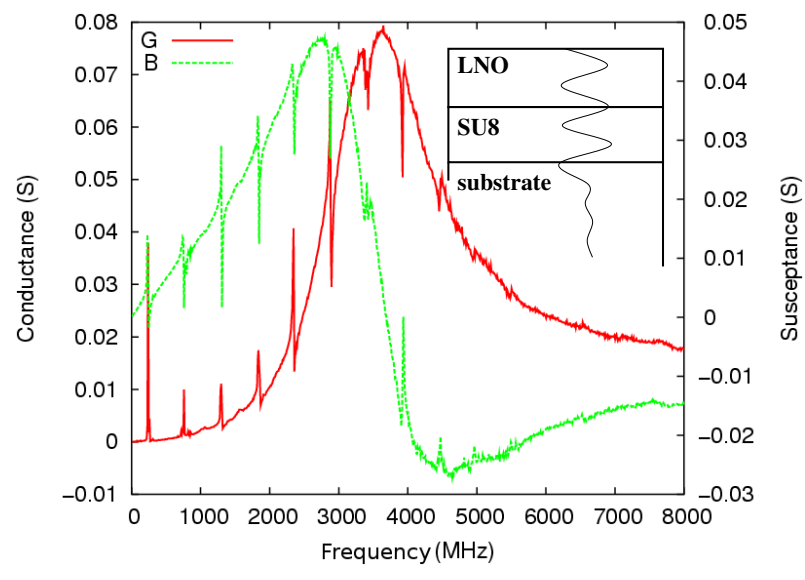

Fig. 6. A stack of two lithium niobate $\mathrm{Y}+36$ glued using a thin SU8 photoresist film exhibits modes up to $4 \mathrm{GHz}$. Top right: schematic of the displacement field in the stack of materials, with the excitation generated by the piezoelectric layer (sandwiched between two electrodes, not shown), the confinement of energy in the thin SU8 film acting as the resonator cavity, with a minute amount of energy reaching the substrate (large impedance mismatch between SU8 and the substrate) which here only acts for strengthening the structure.

\section{CONCLUSION}

We have considered High-overtone Bulk Acoustic Resonators as cooperative targets compatible with a wide range of VHF RADARs, exploiting their broad spectral characteristics. A single transducer is hence compatible with RADAR setups operating in the 100 to $250 \mathrm{MHz}$ range, with little requirements on the hardware available to a user other than a recording capability of over $2 \mu \mathrm{s}$. We have demonstrated such results on a commercially available Ground Penetrating RADAR unit fitted with $250 \mathrm{MHz}$ shielded antennas.

Secondly, we have considered the compatibility of acoustic sensors with microwave RADARs, using as a first approach the microwave carrier modulation in order to load an UHF acoustic sensor with a demodulated response signal lying within the band pass of the sensor. When reaching technology maturity, HBAR is expected to allow to operate in the microwave range and thus to improve efficiency by removing the demodulating diode from the passive sensor.

\section{REFERENCES}

[1] C.S. Hartmann and L.T. Claiborne. Fundamental limitations on reading range of passive IC-based RFID and SAW-based RFID. In 2007 IEEE International Conference on RFID, Gaylord Texan Resort, Grapevine, TX, USA, March 26-28 2007.

[2] D. Rabus, T. Baron, É. Lebrasseur, S. Alzuaga, G. Martin, S. Ballandras, and J.-M. Friedt. Novel narrowband acoustic sensors for sub-ghz wireless measurements. In IEEE Sensors conference, pages 1309-1312, Limerick, Ireland, 2011.

[3] S. Ballandras, T. Baron, E. Lebrasseur, G. Martin, S. Alzuaga, J.-M. Friedt, J.C. Poncot, and C. Guichard. High overtone bulk acoustic resonators built on single crystal stacks for sensors applications. In Proc. of IEEE Sensors, pages 516-519. IEEE, 2011.

[4] J.H. Kuypers, L.M. Reindl, S. Tanaka, and M. Esashi. Maximum accuracy evaluation scheme for wireless SAW delay-line sensors. IEEE Trans. Ultrason. Ferroelectr. Freq. Control., 55(7):16401652,2008

[5] P. Potherat, J.-P. Duranthon, A. Benoit, and F. Lemaître. The long distance measurement: a need measurement. a tool: the ULB radar device. In Rock Slope Stability RSS 2010, 2010.

[6] R. D. Lorenz, C. Elachi, R. D. West, W. T. K. Johnson, M. A. Janssen, M. Moghaddam, G. A. Hamilton, O. Liepack, A. Bunker, L. E. Roth, S. D. Wall, L. Dente, D. Casarano, and F. Posa. Cassini radio detection and ranging (RADAR): Earth and venus observations. Journal of geophysical research, 106(A12):30,27130,279, 2001.

[7] J.-P. Duranthon, L. Effendiantz, and F. Lemaitre. An original methodology for instable landslides monitoring: the microwave radar for distance measurement. the case study of the national road number 91 at the bottom of the rocky slope of séchilienne. In Geoline International Conference, Lyon, France, 2005.

[8] J. R. Riley, A. D. Smith, D. R. Reynolds, A. S. Edwards, J. L. Osborne, I. H. Williams, N. L. Carreck, and G. M. Poppy. Tracking bees with harmonic radar. Nature, 379:29-30, 1996.

[9] M.M. Jatlaoui, F. Chebila, P. Pons, and H. Aubert. Working principle description of the wireless passive EM transduction pressure sensor. The European Physical Journal Applied Physics, 56:13702, October 2011.

[10] J. Conkle. Wireless sensors for gas turbine engines. In Passive Wireless Sensor Tag Workshop. ISA, 2011.

[11] O.J. Gregory, J.R. Conkle, and T.J. Birnbaum. Wireless temperature measurement system and methods of making and using same, 2011.

[12] B. Patrick Motjolopane and R. van Zyl. A review of rectenna models for electromagnetic energy harvesting. Journal of Engineering, Design and Technology, 7(3):282-292, 2009.

[13] M. Arrawatia, M. S. Baghini, and G. Kumar. Rf energy harvesting system from cell towers in $900 \mathrm{mhz}$ band. In 2011 National Conference on Communications (NCC), pages 1-5, 2011.

[14] D. De Vita and G. Iannaccone. Design criteria for the RF section of UHF and microwave passive RFID transponders. IEEE transactions on microwave theory and techniques, 53(9):29782990, 2005. 